\title{
Topology Optimisation of Wireless Sensor Networks
}

\author{
Aye Min Thike ${ }^{1}$, Sergey Lupin ${ }^{1}$, Roustiam Chakirov ${ }^{2}$ and Yuriy Vagapov ${ }^{3, a}$ \\ ${ }^{1}$ National Research University of Electronic Technology, Zelenograd, Moscow, 124498, Russia \\ ${ }^{2}$ Bonn-Rhein-Sieg University of Applied Science, 20 Grantham-Allee, Sankt Augustin, D-53757, Germany \\ ${ }^{3}$ Glyndwr University, Plas Coch, Mold Road, Wrexham, LL11 2AW, UK
}

\begin{abstract}
Wireless sensor networks are widely used in a variety of fields including industrial environments. In case of a clustered network the location of cluster head affects the reliability of the network operation. Finding of the optimum location of the cluster head, therefore, is critical for the design of a network. This paper discusses the optimisation approach, based on the brute force algorithm, in the context of topology optimisation of a cluster structure centralised wireless sensor network. Two examples are given to verify the approach that demonstrate the implementation of the brute force algorithm to find an optimum location of the cluster head.
\end{abstract}

\section{Introduction}

Wireless sensor networks (WSN) have received much attention in the past decade due to advancements in the area of wireless telecommunication and computer engineering. WSNs are widely used in various applications to gather and collect information where monitoring, observation and surveillance are crucial [1]. A wide variety of communications solutions for industrial automation, mobile object tracking, medical monitoring, environmental monitoring etc. are based on WSNs [2].

The main component of WSNs is a sensor node comprising a sensor element, micro-controller, power supply and radio-communication circuit. The sensor nodes designed for industrial applications are embedded into industrial environment in order to provide monitoring of temperature, pressure, vibration, sound and radiation etc. They can also operate as components of industrial automated systems generating information for control algorithms. The size of a modern sensor node is less than a few cubic centimetres [3].

A sensor node is usually a low-power device where data processing through the sensor and microcontroller requires much less energy than radio transmission. Because of this the range of its reliable telecommunication is quite limited. In order to provide a reliable operation of a network WSN should be designed to guarantee stable communication of the sensor nodes at low power consumption. Topology of WSN is a key parameter affecting on provision of reliable communication and, therefore, a crucial part of the network design $[4,5]$.

In an industrial environment the position of a sensor node is often fixed and determined by the particular equipment needs. The WSN topology generated under this circumstance could not support a reliable radio communication between the nodes. To improve commutation reliably the sensor nodes are divided into clusters where the cluster head can provide radio communication with the nodes at a

\footnotetext{
${ }^{a}$ Corresponding author : y.vagapov@glyndwr.ac.uk
} 
longer distance. As an example, Figure 1 demonstrates a concept of a 7-node cluster of centralised WSN.

Finding of the optimum location of a cluster head is the main problem of WSN topology design particularly for an industrial environment. The paper discusses optimisation approach based on the brute force algorithm in context of topology optimisation of a centralised WSN having a cluster structure.

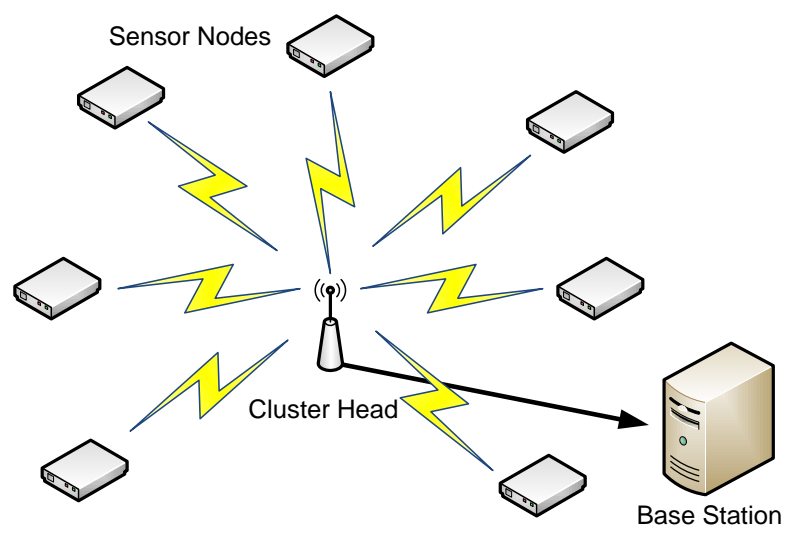

Figure 1. Concept of a cluster of centralised sensor wireless network.

\section{Topology optimisation}

Optimisation problems (including topology optimisation) are often solved using genetic algorithms. These algorithms rely on heuristics based on the principle of natural selection. Genetic algorithms can be successfully applied for a variety of optimisation tasks inappropriate for standard optimisation algorithms, particularly, if an objective function is discontinuous, not differentiable, stochastic or substantially nonlinear. [2, 6, 7] demonstrate examples of successful implementation of the genetic algorithms for various WSN designs. In particular, [7] proposed a genetic algorithm to determine an optimal location of sensor nodes in the network in order to minimise energy consumed by nodes to transmit data. The work also investigated various approaches to the solution including the combining of sensor nodes into clusters to reduce computational complexity of the algorithm. A major disadvantage of genetic algorithms however is related to difficulties of their adaptation to a task formulation.

An alternative approach to optimisation is the introduction of a brute force algorithm. This algorithm is more universal and can be easily implemented with great ease whilst comparing genetic algorithms. However, brute force algorithm is not widely used in optimisation tasks due to high demand in computational resources. Conversely the recent advancements in the area of computing such as multi-core processors and accelerators significantly increased the computing capability of modern computers making brute force algorithm applicable for optimisation task solutions.

Application of the brute force algorithm for WSN topology optimisation can be formulated as following:

1. Let $\left\{\mathbf{A}_{N}\right\}$ be a finite set of network elements which can occupy possible positions $\left\{\mathbf{P}_{M}\right\}$. In practice, the number of positions is much larger than the number of elements: $M>>N$.

2. Find the distribution of elements $\left\{\mathbf{A}_{N}\right\}$ at the positions $\left\{\mathbf{P}_{M}\right\}$ corresponding to an extrema of some functional. In general, the task can be multi-objective.

This formulation of the problem allows for implementation of a brute force algorithm as a multithreaded application where computational complexity of the algorithm is defined as $O\left(M^{N}\right)$. The 
examples below demonstrate of implementation of a brute force algorithm to find the optimum topology for a centralised WSN having one (example 1) and two (example 2) cluster heads.

\section{Topology optimisation criteria}

The optimum criteria for the cluster head allocation can be developed using the following conditions.

1. Power of the communication signal $P_{i}(R)$ is inversely proportional to square of the distance between the cluster head and a sensor node $R$.

$$
P_{i}(R)=P_{i}^{0} / R^{2}
$$

where $P_{i}^{0}$ is the rated power of communication signal of $i$-th sensor node; $R$ is the distance between the cluster head and a sensor node.

2. The communication between the cluster head and a sensor node is possible if a sensor node is located in the area of reliable wireless communication.

$$
R \leq R_{\max }
$$

where $R_{\max }$ is max distance of reliable communication

3. The network consists of a number of sensor nodes $\left\{\mathbf{S}_{k}\right\}$ where each sensor node $S$ is characterised by location coordinates and number transferred parameters into the network $D$.

$$
S_{i} \leq\left(X_{i}, Y_{i}, D_{i}\right)
$$

Therefore the optimisation criteria can be formulated as following:

1. Maximising number of sensor nodes communicating to the cluster head.

$$
F_{1}=\sum_{1}^{K} C\left(S_{i}\right) \rightarrow \max
$$

where $C\left(S_{i}\right)=1$ if the distance between a sensor and any cluster head is less than $R_{\max }$.

2. Maximising the number of parameters transferred by sensors to the network.

$$
F_{2}=\sum_{1}^{K} D\left(S_{i}\right) * C\left(S_{i}\right) \rightarrow \max
$$

where $D\left(S_{i}\right)$ is a number of the object parameters transferring by a sensor node $S_{i}$ to a cluster head.

3. Maximising intensity of communication signal of the cluster head for all sensor nodes.

$$
F_{3}=P_{i}^{0} / R^{2} \rightarrow \max
$$

Computational complexity of the optimisation under above criteria is approximately the same. The given below example of the optimisation utilises the criterion function $F_{3}$.

\section{Examples of cluster head topology optimisation}

In order to verify the proposed method of topology optimisation two examples of a WSN are considered. The model of WSN used in each example consists of 9 identical sensor nodes allocated in a flat industrial environment. The coordinates of the sensor node locations are chosen arbitrary; their details are given in Table 1. The sensor nodes are considered as low-power devices having a limited range of radio communication. The nodes communicate with the cluster head which transfers the information to the base station for further processing. It is assumed that all nodes are operating under a stable clock synchronisation. 
The optimisation problem in the first example is formulated as a determination of the optimum location of the cluster head in order to provide a power saving operation of the nodes. The optimisation criterion is the intensity of the wireless communication signal at the network nodes' locations. It is assumed that the intensity of wireless communication signal is inversely proportional to square of the distance between the cluster head and a sensor node (criterion $F_{3}$ ). The criterion function for each variant of the cluster head allocation is based on sensor having minimum value of the criterion $F_{3}$. The optimal solution should provide the best value of the criterion. It is obvious that solution accuracy depends on the accuracy of the cluster head coordinates representing grid cell size.

Table 1. Coordinates of the sensor nodes.

\begin{tabular}{|c|c|c|}
\hline $\begin{array}{c}\text { Number of } \\
\text { sensor node }\end{array}$ & Axis X & Axis Y \\
\hline 1 & 13.5 & 8.7 \\
\hline 2 & 13.1 & 9.3 \\
\hline 3 & 13.9 & 10.6 \\
\hline 4 & 4.8 & 8.9 \\
\hline 5 & 5.7 & 10.6 \\
\hline 6 & 6.4 & 9.5 \\
\hline 7 & 9.2 & 13.3 \\
\hline 8 & 10.1 & 15.3 \\
\hline 9 & 11.2 & 14.1 \\
\hline
\end{tabular}

The solution for the first example represents the coordinates of the cluster head optimum location. According the optimisation algorithm, the area of flat industrial environment has been represented using a grid layout where the solution coordinates are the centre of a gridded region. The optimisation has been conducted using three grid cell sizes: $2.0,1.0$, and 0.5 . The results of the optimisation are given in Table 2. Figure 2 shows the solution coordinates for three grid cell sizes. It has been found that the accuracy of the solution depends on the grid size. The best (highest) value of the criterion has been obtained for the smallest grid cell size. The reduction in the grid cell sizes however will significantly increase the employment of computational resources and calculation time whereas the grid cell size of 0.5 will provide a satisfactory accuracy.

Table 2. Solution coordinates for the cluster head at various grid cell size.

\begin{tabular}{|c|c|c|c|}
\hline \multirow{2}{*}{ Grid cell size } & \multicolumn{2}{|c|}{ Solution coordinates for the cluster head } & \multirow{2}{*}{ Criterion function } \\
\cline { 2 - 3 } & $\mathrm{X}$ & $\mathrm{Y}$ & \\
\hline 2 & 11 & 9 & 0.0260078 \\
\hline 1 & 10.5 & 8.5 & 0.0306279 \\
\hline 0.5 & 10.25 & 9.25 & 0.0335289 \\
\hline
\end{tabular}

Another example divides the sensor nodes area onto two clusters having two cluster heads. The optimisation problem for the second example is formulated as the coordinates of the optimum location of two cluster heads which form two clusters. The same conditions in terms of the grid cell size have been applied to the second example. The results of the optimisation are given in Table 3. Figure 3 shows the solution coordinates for three grid cell sizes where the sensor nodes are divided onto clusters. As in the previous example, the best (highest) value of the criterion has been obtained for the smallest grid cell size. It should be noted that the algorithm generates two solutions for each pair of the cluster heads. In fact two solutions are identical representing two symmetrical coordinates for the cluster heads. 


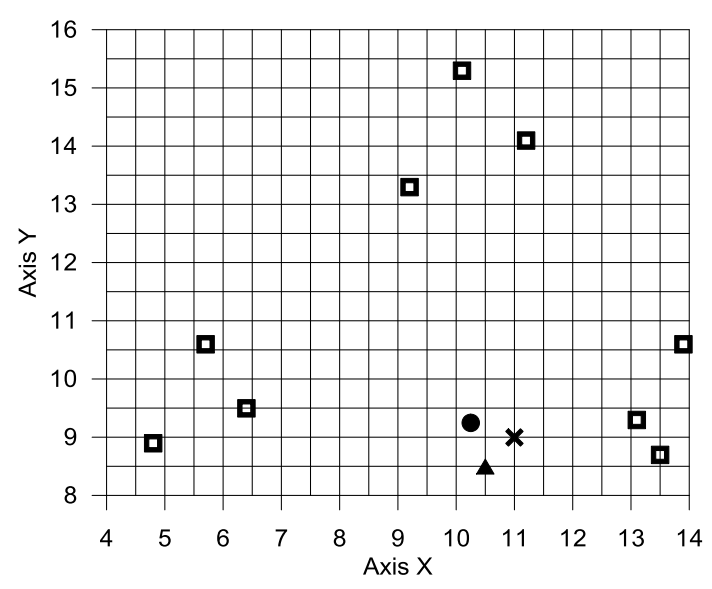

Figure 2. Solution coordinates of one cluster head, where $\square-$ sensor nodes, $\mathbf{x}$ - the solution coordinates of the cluster head at grid cell size of $2.0, \boldsymbol{\Delta}$ - the solution coordinates of the cluster head at grid cell size of $1.0, \bullet-$ the solution coordinates of the cluster head at grid cell size of 0.5 .

Table 3. Solution coordinates for two cluster heads at various grid cell size.

\begin{tabular}{|c|c|c|c|c|c|}
\hline \multirow{2}{*}{ Grid cell size } & \multicolumn{4}{|l|}{ Solution coordinates for two cluster heads } & \multirow{2}{*}{ Criterion function } \\
\cline { 2 - 5 } & $\mathrm{X}_{1}$ & $\mathrm{Y}_{1}$ & $\mathrm{X}_{2}$ & $\mathrm{Y}_{2}$ & \\
\hline 2 & 7 & 11 & 13 & 9 & 0.0729927 \\
\hline 2 & 13 & 9 & 7 & 11 & 0.0729927 \\
\hline 1 & 6.5 & 11.5 & 12.5 & 9.5 & 0.0735294 \\
\hline 1 & 12.5 & 9.5 & 6.5 & 11.5 & 0.0735294 \\
\hline 0.5 & 6.75 & 11.75 & 12.75 & 8.75 & 0.079968 \\
\hline 0.5 & 12.75 & 8.75 & 6.75 & 11.75 & 0.079968 \\
\hline 0.25 & 7.125 & 11.375 & 12.625 & 9.125 & 0.0867209 \\
\hline 0.25 & 12.625 & 9.125 & 7.125 & 11.375 & 0.0867209 \\
\hline
\end{tabular}

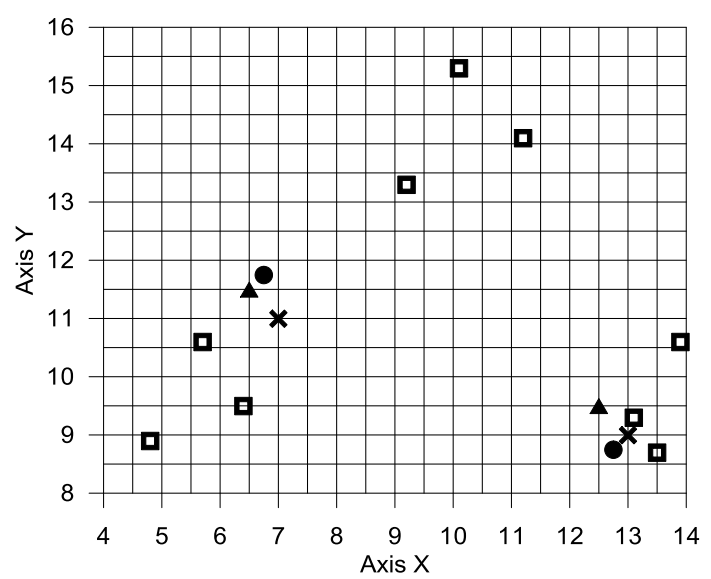

Figure 3. Solution coordinates of two cluster heads, where $\square-$ sensor nodes, $\mathbf{x}-$ the solution coordinates of the cluster heads at grid cell size of $2.0, \boldsymbol{\Delta}$ - the solution coordinates of the cluster heads at grid cell size of $1.0, \bullet-$ the solution coordinates of the cluster heads at grid cell size of 0.5 . 


\section{Conclusion}

The examples demonstrate an effective implementation of the brute force algorithm to determine the optimum location of the cluster heads in WSNs where the optimisation criterion is intensity of radio signal at the network nodes' locations. The algorithm is invariant with respect to the criterion function and can be used for optimisation under another criterion without substantial modifications. The proposed algorithm was used for the location optimisation on a flat area. The further work is related to implementation of the algorithm for topology optimisation in a three dimensional space area.

A significant drawback of the proposed method is a high computational complexity. For example, the solution for two cluster heads under the grid cell size of 0.5 required a search time of 150 seconds. For relatively small numbers of cluster heads and sensor nodes the solution can be calculated using a powerful workstation. However for large values the solution computational algorithm should be executed under a multi-threaded approach using, for example, Intel Xeon Phi accelerators. Another approach to reduce computational complexity is based on a topology fragmentation. Under this approach the solution can be found as a set of optimum solutions obtained for a number of local topologies. The further work aims to investigate implementation of topology fragmentation algorithms for topology optimisation of WSNs.

The paper has been supported by RFBR No 16-07-01055\165 "Adaptation of resource demanding algorithms to distributed computational environment".

\section{References}

1. P.-J. Chuang, Y.-J. Jiang, Effective neural network-based node localisation scheme for wireless sensor networks, IET Wireless Sensor Systems, 4(2), 97-103 (2014)

2. N. Panigrahi, P.M. Khumar, Optimal topological balancing strategy for performance optimisation of consensus-based clock synchronisation protocols in wireless sensor networks: A genetic algorithm-based approach, IET Wireless Sensor Systems, 4(4), 213-222 (2014)

3. X. Shen, Z. Wang, Y. Sun, Wireless sensor networks for industrial applications, 5th World Congress on Intelligent Control and Automation, 3636-3640 (2004)

4. L F. Ge, Y. Wang, An adaptive energy efficient topology for wireless sensor networks, 2nd Int. Conf. on Future Generation Communication and Networking, 178-192 (2008)

5. H. Chen, C.K. Tse, J. Feng, Impact of topology on performance and energy efficiency in wireless sensor networks for source extraction, IEEE Trans. on Parallel and Distributed Systems, 20(6), 886-897 (2009)

6. C. Charalambous, S. Cui, A biologically inspired networking model for wireless sensor networks, IEEE Network, 24(3), 6-13 (2010)

7. A.P. Bhondekar, V. Renu, M. Singla, C. Ghanshyam, P. Kapur. Genetic algorithm based node placement methodology for wireless sensor networks, Int. Multi Conf. of Engineers and Computer Scientists, 1, 106-112 (2009) 